\title{
New Insights into the Structure of PtPd Bimetallic Nanoparticles and Their Atomic Resolution Images by Cs-Corrected STEM
}

\author{
Subarna Khanal, Nabraj Bhattarai, Gilberto Casillas, J. Jesus Velazquez-Salazar, and Miguel Jose- \\ Yacaman
}

Department of Physics and Astronomy University of Texas at San Antonio, One UTSA Circle, San Antonio, Texas, 78249

The chemical synthesis and morphological control of bimetallic (BM) nanoparticles has received particular interest because of their myriad properties and diverse potential applications such as: optical, magnetic, catalysis and others, which are highly tunable and superior compared with those of their monometallic one. [1,2] The fabrication of such BM core-shell, heterostructures, and alloy nanocrystals are the possible candidate for increasing mass specific activity of the nanoparticle and also minimizing the cost by decorating the precious metals in the shell. Moreover, the purpose of better tailoring their activities and understanding the correlation between their structures and various properties, control over the shape, size and composition. The distinct feathers expected for these BM nanostructures include the physical and chemical interactions among different atoms and phases that lead to novel functions, the changed miscibility and interactions unique to nanometer dimensions, and the morphological variations that are related to new particles.

Transmission electron microscopy (TEM) and High Resolution TEM JEOL-2010F operated by 200kV equipped with field emission gun and an ultra-high-resolution pole piece was employed to study the morphology, high resolution images and nano-beam diffraction. The STEM images were recorded in probe Cs-corrected JEOL JEM-ARM 200F operated at $200 \mathrm{kV}$. HAADF STEM images were obtained with a convergence angle of $26 \mathrm{mrad}$ and the collection semi-angles from 50 to $180 \mathrm{mrad}$. These variations in semi-angles satisfy the conditions set forth for the detectors to eliminate contributions from unscattered and low-angle scattered electron beams. The probe size used was about $0.09 \mathrm{~nm}$ with the probe current of $22 \mathrm{pA}$. In addition, bright field (BF) STEM images were recorded by using a collection semi-angle of $11 \mathrm{mrad}$. Electron dispersive $\mathrm{x}$-ray spectroscopy (EDS) spectra were obtained using a probe size of $0.13 \mathrm{~nm}$ with the probe current $86 \mathrm{pA}$.

In this present work, we have successfully synthesized Pt-Pd core-shell nanoparticles via a modified polyol methods. The overgrowth of the thin Pd multishell on the Pt cores due to the epitaxial growth modes was observed. We predicted that the truncated octahedral Pt-Pd core-shell nanocrystals were formed in both FM and SK growth modes. Moreover, the surface of the nanoparticle is not perfectly smooth, and contains step edges and kink sites that are active for chemical reactions. The relevant effect in the shell shape of this type of core-shell nanoparticles is the internal strain caused by the lattice mismatch between Pt and Pd (0.77\%). The structural defects such as stacking faults are also observed into Pt-Pd core-shell nanocrystals surfaces. Thus, the HRTEM and $\mathrm{C}_{\mathrm{s}}$-corrected HAADF-STEM images revealed that the structural and chemical features of the Pt-Pd core-shell nanoparticles may lead to development of structurally stable and chemically active nanocrystal catalysts for various applications.

\section{References}

[1] R. Ferrando, J. Jellinek and R. L. Johnston, Chemical Reviews, 2008, 108, 845. 
[2] B. Lim, et al., Science, 2009. 324, 1302.

[3] S. Khanal, et al., Journal of Physical Chemistry C, 2012, 116, 23596.

[4] Acknowledgments: The authors would like to acknowledge NSF grants DMR-1103730, “Alloys at the Nanoscale: The Case of Nanoparticles Second Phase”, PREM: NSF PREM Grant \# DMR 0934218, and Welch Foundation (grant No. AX-1615).
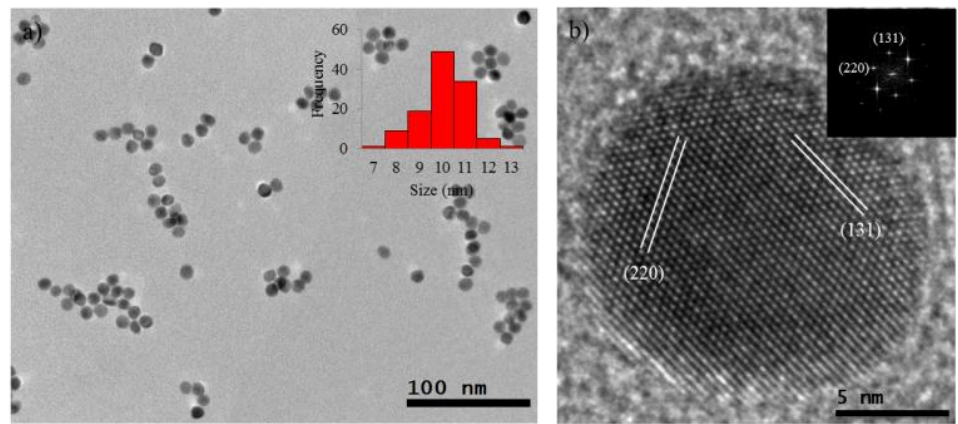

Figure 1. (a) TEM image Pt nanoparticles. (b) HRTEM image of a Pt seed in a [114] zone axis where the (220) and (131) lattice planes can be observed.
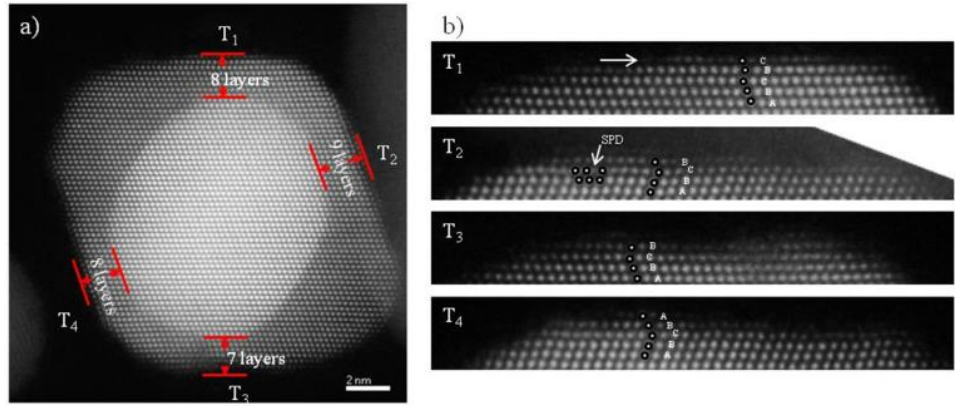

Figure 2. (a) HAADF image of an octahedral core-shell nanoparticles in a [011] zone axis. (b) Closeups of the (111) surfaces where the defects are readily observed.
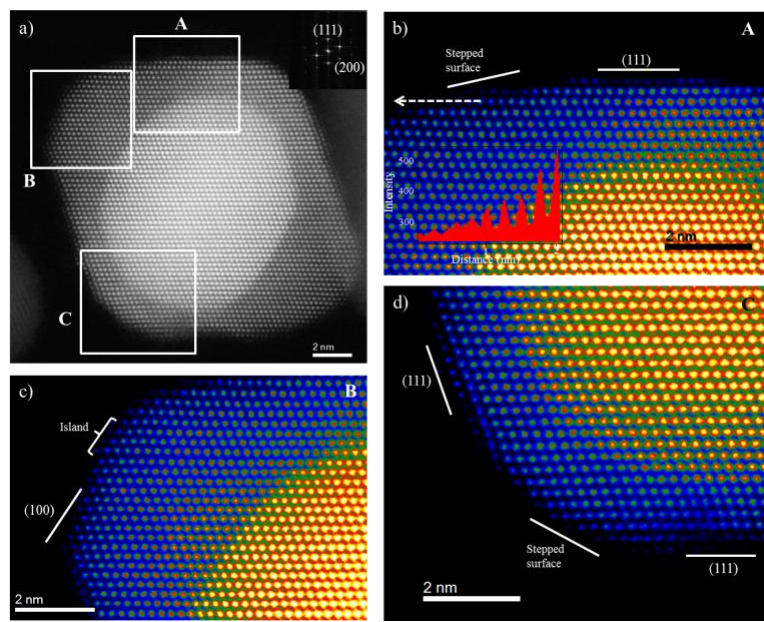

Figure 8. (a) HAADF-STEM image of a Pt-Pd core-shell truncated octahedral nanoparticles. (b) STEM image (square area A) near (111) surface plane with kinks. The inset intensity profile along the dotted arrow represents a stepped surface. (c) Magnifying image from area B in (a). In that truncated region, a monoatomic layer island on the (100) plane can be observed. (d) Zoom in image from area C in (a). Here, the octahedral corner exhibits terraces and stepped surface along the edge near (111) surface planes. 